\title{
El agroturismo una oportunidad de desarrollo para la sierra de Sonora
}

\author{
Santiago-Hernández Víctor Guadalupe ${ }^{*}$, Alcaraz-Miranda Martina², Córdova-Yánez Alejandro³ \\ 1. Responsable de CAEF: Desarrollo Regional Sustentable. PTC UNISIERRA. \\ 2. PTC UNISIERRA \\ 3. Jefe de DCEA
}

\section{Resumen}

Cuando la tierra no solo produce bienes, sino servicios es porque la diversificación está en proceso; precisamente, es aquí cuando la forma de pensar de los productores se modifica en beneficio de su familia, sino de su entorno social y económico.

Los productores de la región serrana del estado de Sonora, la cual se comprende para el presente estudio 18 municipios, es caracterizada esencialmente por la práctica de actividades agropecuarias, esta cultura productiva ha incitado un prototipo socioeconómico que históricamente radica en el quehacer cotidiano de las comunidades, el cual no ha permitido en su totalidad la diversificación de las actividades económicas en dicha región.

Haciendo referencia a la perspectiva del productor en la región, la disposición es escasa y nula por parte de los propietarios de ranchos para transformar o diversificar sus actividades cotidianas, debido a que no consideran visualizar las posibilidades de desarrollo en su entorno, haciendo una incorporación de actividades en relación particularmente con el agroturismo.

Dicho lo anterior, se espera que con esta investigación se pueda concienciar a los productores que vale la pena modificar su enfoque hacia la elaboración de hacer tangible el turismo en experiencias memorables.

Esta investigación es resultado del trabajo colegiado del Cuerpo Académico Desarrollo Regional Sustentable, el cual está enfocado en analizar, promover y difundir la actividad económica en la sierra de Sonora.

Palabras clave: Agroturismo, sierra, economía, productores, Sonora.

\section{Agrotourism a development opportunity for the Sierra de Sonora}

When the earth shares not only goods, but services is because diversification is in process; precisely, here is when the way of thinking of the producers reforms for the benefit of his family, but its social and economic environment.

The producers of the mountainous region of the Sonora`s State, which comprises 18 municipalities for this research, is essentially characterized by the practice of farming, this productive culture has prompted a socioeconomic prototype historically lies in the everyday activities of the communities, which has not allowed the diversification of economic activities in the region in its entirety.

With reference to the perspective of the producers in the region, the provision is scarce and null by the owners of farmers for transform diversify their everyday activities, since they consider does not view the possibilities of development in its environment, particularly by an incorporation of activities in relation with agrotourism.

That said, is expected with this research to educate producers worth to modify its approach to the development of tourism in memorable experiences make tangible.

This research is the result of the collegiate work academic called Development Regional Sustainable, which is focused on, promoting and disseminating the economic activity in the mountains region of the state of Sonora.

Keywords: Agrotourism, mountains, economy, producers, Sonora. 


\section{INTRODUCCIÓN}

En la actualidad, el limitado crecimiento económico registrado en la región de estudio derivado de la práctica de la ganadería, considerada esta, como una las fuentes del sector primario en ingresos para la región, propicia el análisis propositivo de ideas alternativas de desarrollo local, como herramientas para analizar actividades que detonen la región serrana, contando con todos aquellos recursos históricos, culturales y naturales, que puedan diversificar las alternativas a desarrollar y desde el enfoque de colaboración, impacten de manera significativa en la mejora de la calidad de vida de los habitantes.

La realidad anteriormente expuesta, se puede ver reflejada con datos del Instituto Nacional de Estadística, Geografía e Informática (INEGI), no es una situación única que enfrenta la región de estudio, en el estado de Sonora se observa un constante crecimiento de los sectores económicos secundarios y terciarios, y como consecuencia de esta realidad, se registra una disminución significativa del sector económico primario, a continuación la Tabla 1, muestra el porcentaje de las actividades con aportación al Producto Interno Bruto (PIB) para el estado de Sonora en el año de 2014, con cifras preliminares, y estas a su vez evidencian las actividades más relevantes de esta parte del noroeste de México.

Tabla 1. Actividades económicas de Sonora
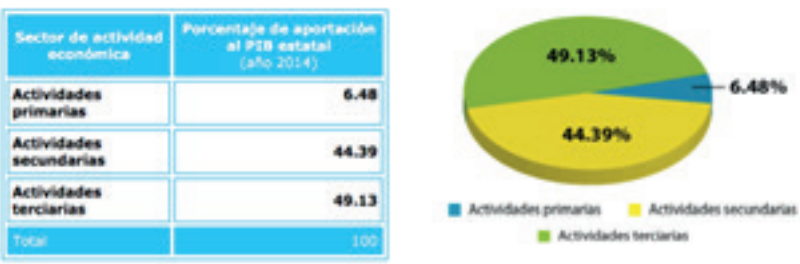

Fuente: INEGI. Sistemas de Cuentas Nacionales de México, 2008. Participación por actividad económica, en valores corrientes, 2014.

La estructura limitada de este crecimiento socioeconómico en la región, también ha impactado en la estabilidad social y económica de los 18 municipios que comprenden la región en cuestión, (Arivechi, Bacadéhuachi, Bacanora, Bacerac, Bavispe, Cumpas, Divisaderos, Fronteras, Granados, Huachinera, Huásabas, Moctezuma, Nácori Chico, Nacozari de García, Sahuaripa, San Pedro de la Cueva, Tepache y Villa Hidalgo) estas incidencias han generado de forma indirecta una migración interna del estado, propiciando un tránsito de las zonas o regiones rurales a las urbanas y por consecuencia, un abandono de actividades directamente vinculadas al sector productivo primario.

De acuerdo con datos del INEGI, (2010), el porcentaje de personas que habitan en comunidades rurales ha disminuido. Para 1950, representaba poco más del 43\% del país, en el año de 1990 era de un $81 \%$, y 20 años después, esta cifra cambió a un $78 \%$, esta muestra representativa de la realidad del país no es lejana de la presentada por el estado de Sonora y particularmente de la región sierra.

Como parte de las políticas públicas desarrolladas por los gobiernos estatales en los periodos (2003-2009) y (20092015), la inclusión de desarrollo socioeconómico para la región sierra, ha considerado la realidad anteriormente descrita y argumentada por los datos de INEGI (2010), y con ello ha diseñado estrategias enfocadas al apoyo de nichos diversificados de las actividades primarias naturales de la región, permitiendo que actividades secundarias y terciarias especializadas en servicios, reciban mayor impulso.

En los últimos 10 años, el fomento, promoción y diversificación a actividades de turismo rural en la región sierra, han representado una opción de diversificación de actividades agrícolas y no agrícolas en beneficio de los propietarios, miembros de la comunidad receptora, a través de nuevas fuentes de empleo, ingresos rurales, y argumentos para permanecer en los espacios rurales (Ascanio, 2009).

Según la Organización Mundial del Turismo (OMT) a través de la Agenda de Innovación de Sonora (2014), México ocupó en 2003 el lugar número ocho por su importancia turística a nivel global, sin embargo, en el 2013 el país retrocedió cinco lugares y volvió a tomar la posición que tuvo en los años 50's.

Lo anterior advierte, que no se ha intervenido el interés por optar alternativas de atraer turistas a la región serrana del estado de Sonora, desde una visión estructurada y asociada por actores del desarrollo socioeconómico institucional, empresarial y civil. El motivo fehaciente de la realización del análisis contextual actual, sobre la perspectiva de productores y turistas, gracias a la información obtenida por medio de entrevistas estructuradas, permiten al estudio conocer en un primer momento el comportamiento de turistas e identificar el conocimiento por parte de los propietarios de ranchos, acerca del tema referente al agroturismo.

El enfoque que se le está dando al turismo alternativo, en especial al agroturismo, en la región serrana del estado de Sonora, es tal que pueda manifestarse como la posibilidad de insertarse en las actividades cotidianas del productor y la dinámica socioeconómica de las comunidades, accediendo de esta manera la creación de un micro-clúster productivo, como una estrategia alterna a los sistemas individuales o de colaboración escaza existente. Para la realización de lo anteriormente mencionado, es preciso indicar los municipios considerados como parte del trabajo realizado, 
(véase Figura 2).

Figura 2

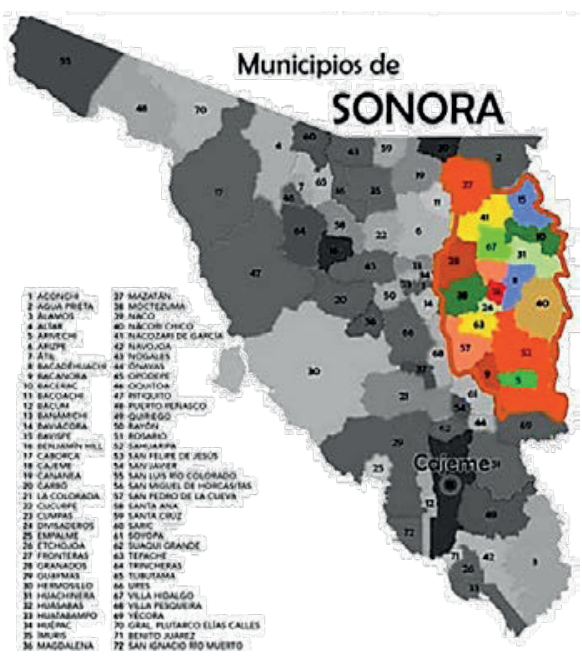

Fuente: Elaboración propia, 2018

La realidad de la región sierra, es escenificada por un escaso desarrollo económico, en contraste a esta región, es la realidad de otros territorios (regiones) en el estado, que generaron una visión de desarrollo turístico, a su dinámica socioeconómica, tal es el caso del Pueblo Mágico de Álamos en la región sur del Estado de Sonora, al igual que las costas que detonan el turismo binomio (sol y playa) en los municipios de Guaymas y Puerto Peñasco. Si bien, estas condiciones existentes en el resto del estado, propicia el crear y fomentar estrategias diversificadas del turismo, la región sierra, también estructura en sus condiciones geográficas y socioeconómicas, los elementos mínimos necesarias para generar la condiciones de desarrollo agroturístico en la región, como una alternativa para la diversificación de las actividades tradicionales, las cuales, permitan inicialmente detonar el turismo relacionado con el sector agropecuario, como medio para incitar el flujo de esta actividad en el noreste del estado de Sonora.

La importancia del agroturismo radica en apostarle desde el sector primario hasta su conversión y aprovechamiento al de servicios, como una propuesta viable y factible que representa como alternativa de desarrollo local, tanto para los propietarios de ranchos, mismos que son los que cuentan con el recurso, así como a los prestadores de servicios para complementar el clúster turístico que se desea proponer al interior de cada municipio y región.

No hay que perder de vista, que la actividad de relevancia y área de oportunidad para la región sierra, es sin duda alguna el agroturismo, es que se deben buscar implementar una serie de acciones encaminadas a establecer una estrecha relación entre los que poseen el recurso (propietario de ranchos) y estimular la visión de diversificación socioeconómica al área específicamente del agroturismo.

Llevar a cabo un modelo que respalde las ideas de crear lazos con todo aquel propietario de rancho que se catalogue como participante del fomento para un clúster en la región sierra, es con motivo de mantener reforzada la región considerando las ventajas de sus actividades primarias, para la realidad de esta parte de Sonora la vía necesaria es un clúster como medio idóneo que propicie un desarrollo socioeconómico en la región. La productividad se hace evidente entre las empresas que están establecidas, esta a su vez significa la innovación de nuevos procesos para la diversificación de las actividades primarias dándoles un atractivo que incite la afluencia turística, por lo tanto, esto promueve nuevos empleos.

El objetivo general de la presente investigación es proyectar la potencialidad que tienen los productores frente al agroturismo, para la detonación y diversificación de sus actividades económicas, así como la reconversión de actividades productivas. Para ello, se han definido los siguientes objetivos específicos: identificar las características que tienen los productores de la región serrana del estado de Sonora, mediante la aplicación de una encuesta donde se permeen las actitudes que ayudaron a medirlo; segundo: analizar las ventajas y condiciones contextuales de los municipios estudiados en la región de la Sierra y; tercero: distinguir los recursos turísticos naturales, y culturales de la región serrana de Sonora.

Como se mencionó anteriormente, este trabajo es un producto de un trabajo colegiado, el cual, en primera instancia se ha provisto de elementos tangibles para poder cimentar estrategias de acción clave para cumplir con los objetivos que tiene el Cuerpo Académico denominado: Desarrollo Regional Sustentable.

El estado de Sonora, no debería quedar fuera de las oportunidades de desarrollo en cuanto al turismo rural de refiere del desarrollo del turismo rural, ya que a nivel nacional vía SECTUR (Secretaría de Turismo) y, a nivel estatal por COFETUR (Comisión de Fomento al Turismo), han buscado fortalecer no solo el turismo convencional de sol y playa, sino el que es propio de regiones con un sentimiento de arraigo y cultural.

En el proceso de este trabajo de investigación, se mencionan algunas definiciones pertinentes al tema, iniciando con la denominación turismo rural está relacionada a la motivación del viaje. Según la Organización Mundial del Turismo, el turismo es el motivo del desplazamiento lo que caracterizará 
la tipología turística. Así, el turismo rural es definido como aquel en el cual el motivo principal del viaje es conocer las actividades del mundo rural (OMT 2001 citado en Henríquez, Zechner y Cioce, 2010, p. 24).

El turismo rural, y en especial el agroturismo, es considerado por las autoridades públicas y privadas como una estrategia para dinamizar el desarrollo de las zonas rurales por su capacidad de contribuir a la generación de ingresos adicionales a los productores agropecuarios (Blanco y Riveros, 2011 citado en Apodaca-González et al., 2014, p.1526).

Para los productores agropecuarios, el turismo rural es una actividad que se erige como una alternativa económica, complemento de su producción, que permite el uso de la capacidad instalada ociosa de los establecimientos, así como también la inclusión de la mano de obra familiar no ocupada en la producción tradicional, principalmente la de mujeres y jóvenes.

Es preciso distinguir las tres categorías dentro del turismo alternativo, estas con ramificaciones específicas en su área: turismo alternativo, ecoturismo y turismo Rural (de esta deriva el agroturismo) cabe hacer mención a lo que turismo rural se refiere. El concepto de turismo rural se le considera como "el lado más humano del turismo alternativo" (Ascanio, 2009, p.13).

Dado que esta investigación se refiera al agroturismo, la Organización Mundial del Turismo (1996), define el agroturismo de la manera siguiente: el que se realiza en explotaciones agrarias (granjas o plantaciones) que complementan sus ingresos con alguna forma de turismo, facilitando por lo general hospedaje, alimentos y oportunidad de familiarización con trabajos agropecuarios.

El agroturismo, se entiende como la modalidad turística en áreas agropecuarias, con el aprovechamiento de un medio ambiente rural, ocupado por una sociedad campesina, que muestra y comparte no solo su idiosincrasia y técnicas agrícolas, sino también su entorno natural en conservación, las manifestaciones culturales y socio productivas, en donde se busca que la actividad represente una alternativa para lograr que el campesino se beneficie con la expansión de su actividad económica, mediante la combinación de la agricultura y el turismo (Secretaría de Turismo, 2004).

El turista comienza a mirar hacia un turismo alternativo, flexible y heterogéneo, con cierta sensibilidad hacia aspectos medioambientales, $y$ de experiencias diferenciadas e individualizadas, con acercamiento a la cultura local.

\section{MATERIALES Y MÉTODOS}

El tipo de estudio seleccionado, de acuerdo con el método de análisis propuesto para la realización del proyecto de investigación, fue del tipo descriptivo. Este tipo de estudio de acuerdo con el autor Hernández Sampieri, et al (2014) buscan especificar las propiedades, las características y los perfiles de las personas, grupos, comunidades, procesos, objetos o cualquier otro fenómeno, que se someta a un análisis.

El enfoque del proceso de investigación, se realizó desde una perspectiva mixta, debido a que esta, implica un proceso de recolección de datos de tipo cuantitativos y cualitativos, específicamente desde la realidad de este estudio los datos provenientes de propietarios de ranchos, los cuales posteriormente, fueron sujetos a un análisis de información sobre su percepción sobre el contexto de estudio, así como de sus apreciaciones de los recursos y condiciones de los mismos. "La meta de la investigación mixta, no es reemplazar a la investigación cuantitativa ni a la investigación cualitativa, sino utilizar las fortalezas de ambos tipos de indagación, combinándolas y tratando de minimizar sus debilidades potenciales".

La encuesta fue aplicada a propietarios de ranchos, los cuales fueron elegidos por un muestreo no probabilístico. Dichas recomendaciones fueron iniciadas con el Presidente Municipal, el Presidente Ejidal o en su caso, del Presidente de Productores. El número de encuestas fue de 103 encuestas. Es relevante indicar, que la entrevista a propietarios de ranchos consta de 16 variables a analizar. Además, se utilizó la entrevista semiestructurada para recabar información, tuvo como propósito dar un diagnóstico de las posibilidades de adaptar esta forma de turismo, condicionándolo a los recursos existentes en el noreste del Estado.

Ambos instrumentos, fueron previamente elaborados con la finalidad que cada ítem, amplíe un horizonte de posibilidades que den píe a fomentar el agroturismo y, por ende, persuada a turistas en su percepción de su estadía en la Región de la Sierra de Sonora. El instrumento principalmente utilizado para el proceso de recolección de información, ha sido previamente elaborado, por el cuerpo académico conformado de la Universidad Autónoma de Baja California (UABC, Campus San Quintín), como parte de un proyecto nacional del Programa para el Desarrollo Profesional Docente (PRODEP).

El instrumento presenta una escala de respuesta fundamentada en la escala de Likert, la cual se describe como un sistema de nivel ordinal y se caracteriza por ubicar una serie de frases seleccionadas en una escala con grados de acuerdo/desacuerdo. 


\section{RESULTADOS Y DISCUSIONES}

En este apartado se exponen y describen los hallazgos más relevantes de la aplicación metodológica hacia el agroturismo para la región Sierra de Sonora, para ello se consideró necesaria la interpretación de los gráficos siguientes, describiendo inicialmente el instrumento aplicado a los propietarios de ranchos.

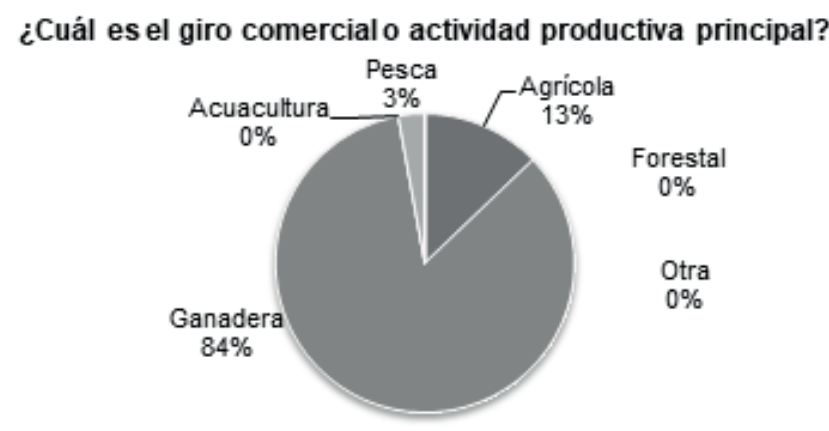

Gráfica 1. Giro comercial, Fuente: Elaboración: propia, 2018.

Con un índice de más del 84\% la región serrana aporta posibilidades de ser impulsada por el turismo, específicamente por el turismo siendo este el recurso en gran extensión existente. En contraste con el porcentaje a nivel estatal respecto a las actividades primarias es alta y diversificada la proporción. Véase, arriba, tabla 1. (Actividades económicas en Sonora). Anteriormente, gráfica representativa de las actividades llevadas a cabo por propietarios de ranchos de los 18 diferentes municipios de la región Sierra del Estado de Sonora.

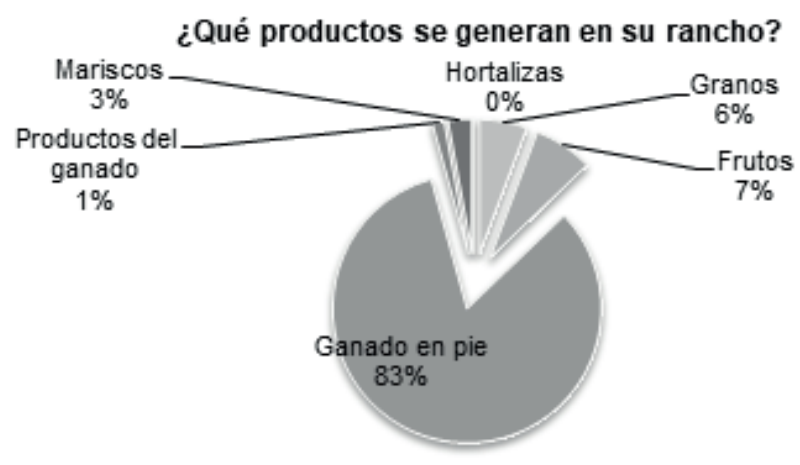

Gráfica 2. Producto generado, Fuente: Elaboración propia, 2018

Es preciso conocer la producción generada en los predios de los propietarios de ranchos que han colaborado con el proyecto de investigación, es decir, conociendo dicho dato otorga un panorama explícito para argumentar el clúster turístico, más de las tres cuartas partes de la producción de los predios respectivos a la región serrana del estado de Sonora acierta un número significativo en la producción de ganado en pie (comercialización de ganado vivo).

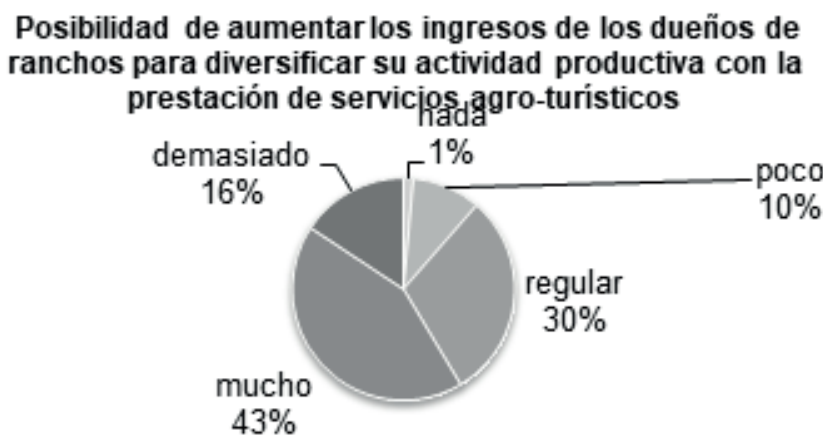

Gráfica 3. Diversificación de actividades

Fuente: Elaboración propia, 2018.

El gráfico anterior, pronostica la posibilidad de ejecutar o no el clúster turístico que abra paso a nuevas tendencias de turismo para la sierra de Sonora conformado por 18 municipios prósperos en recursos endógenos agrícolas, el $43 \%$ de los propietarios de ranchos consideran favorable el incluir esta nueva tendencia de turismo como alternativa de diversificación.

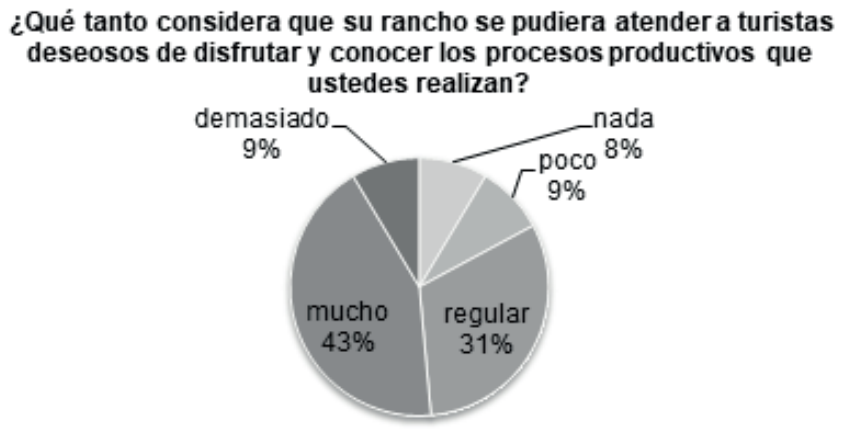

Fuente: Elaboración: propia, 2018.

Gráfica 4. Capacidad de infraestructura

Contemplando la afirmación o negación por parte del propietario de rancho, la capacidad del predio es indispensable tenerlo en consideración, observando el grafico anterior es positiva la posibilidad en un $43 \%$ de acoger turistas interesados en disfrutar y conocer los procesos productivos.

¿Qué tanto le gustaría diseñar recorridos y conducir al turista por atractivos vivenciales dentro de la granja que los hagan aprender y causarles emociones?

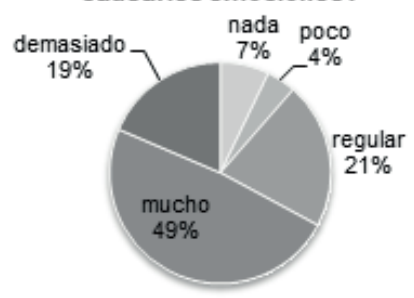

Gráfica 5. Diseño de senderos, Fuente: Elaboración: propia, 2018. 
Parte del agroturismo y las prácticas del mismo, pero sobre todo, por ser una ramificación del turismo rural, turismo rural "el lado más humano del Turismo Alternativo" (Ascanio, 2009, p.13) hace referencia a la convivencia directa con la naturaleza, por ende, el tener senderos, recorridos por los cuales los turistas contemplen los paisajes, enriquece las actividades que se les proporcionen, 49\% de los propietarios aseguran estar dispuestos a hacer recorridos, es decir, senderos por el cual los turistas vivan experiencias de aprendizaje paisajístico.

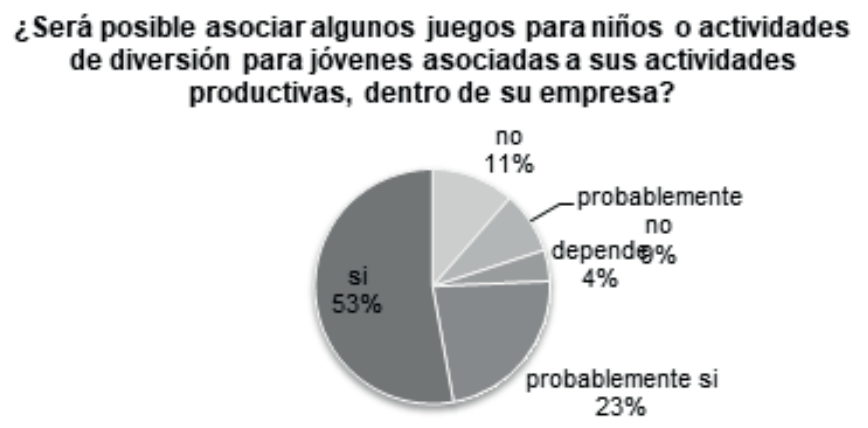

Gráfica 6. Anexo de juegos recreativos, Fuente: Elaboración: propia, 2018.

En promedio más de la mitad considera la posibilidad de incluir juegos productivos de recreación dentro del predio, con un porcentaje por arriba de la mitad, la estadística infiere que hay interés por parte de los propietarios para anexar todo aquel instrumento de recreación para niños y jóvenes que incurran en las experiencias turísticas.

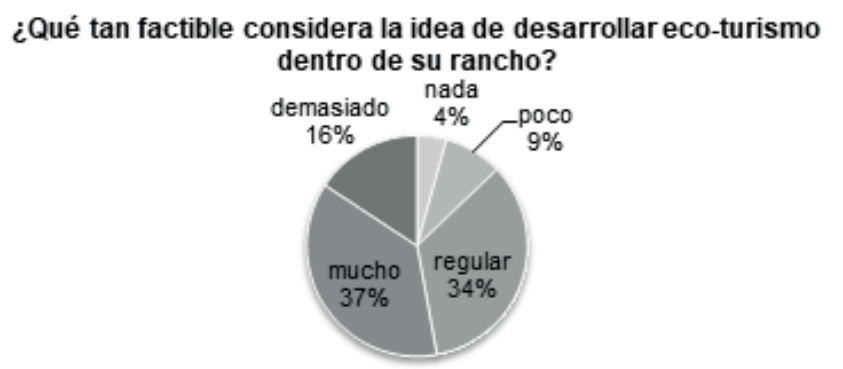

Gráfica 7. Desarrollo de eco-turismo, Fuente: Elaboración: propia, 2018.

El 53\% de los propietarios de ranchos que han sido encuestados aseguran desarrollar turismo dentro de su rancho como idea factible de diversificación de actividades. El rango de aceptación es favorable debido a referencias de aplicación de eco-turismo que han repercutido de forma favorable, y conscientes de ello afirman considerar la idea a desarrollar.

Derivado de lo anterior, se puede argumentar que los resultados están dando la pauta para implementar acciones en curso, los cuales van a permitir que cada microrregión pueda ser aprovechada de forma económica, administrativa y turísticamente hablando.

Cabe señalar, que una de las primeras percepciones sobre las conclusiones iniciales de este apartado, están centradas en los datos analizados del instrumento utilizado, resaltando principalmente la percepción que propietarios de ranchos y turistas (nacionales y extranjeros) tienen acerca del agroturismo, las cuales se consideran aceptables para la incorporación de programas y modelos a las condiciones con las que cuenta cada municipio, los cuales formaron parte de trabajo de campo.

Es a considerar de igual forma con los datos significativos obtenidos de la encuesta estructurada y observación participativa, que la apreciación principalmente de los productores es limitada en la mayoría de los casos documentados, respecto a la incorporación de nuevas alternativas a su dinámica socioeconómica actual y tradicional, por lo cual es necesaria la inclusión de una estrategia, de colaboración y generación de confianza en emprendedurismo, con los recursos y ventajas existentes en sus contextos.

Desde esta primera idea, se considera que el agroturismo sí podrá convertirse en una tendencia en el cual el turista se interese en participar, debido a que el 35\% de los turistas nacionales y el $40 \%$ de turistas internacionales manifiestan una cercanía a vivir experiencias en estos entornos rurales y espacios abiertos (ranchos). Tomando en consideración estas realidades, es viable proceder con la estrategia propuesta de micro-clúster, debido, a la demanda y condiciones existentes presentadas por los productores, municipios, infraestructura y prestadores de servicios.

El estudio demuestra que, en efecto, sí existen las condiciones mínimas para hacer viable técnicamente hablando la propuesta de micro-clúster, en resumen, hay existencia de recursos naturales, aprobación de los propietarios, interés del turista, hay infraestructura, tanto vial, como de hospedaje y alimentación, por lo cual se concluye, la posible ejecución de la alternativa planteada, debido a la positividad que muestra el escenario estructurado, así como la posibilidad de operacionalizar cada uno de los microclúster en la región de estudio.

De lo anterior se deduce que el conocimiento de cada región es relevante para poder tomar decisiones viables de intercambio económico, derivado del hecho de que conociendo y analizando las diversas variables, se puede formalizar actividades propias a cada micro clúster por sector. Estos dan la pauta para hacer propuestas de crecimiento en los ranchos de los productos de la región sierra de Sonora 


\section{CONCLUSIONES}

Es importante mencionar que existen características propias que identifican a cada región, y que la detonación turística de dicho lugar no debe actuar en menoscabo de las diferentes actividades económicas existentes. Por lo tanto, el turismo debe aprovechar y fortalecer la identidad local y así transformar algunos servicios como herramientas para detonar y generar nuevas fuentes de empleo

De acuerdo a lo previsto por este análisis, son diversos los factores que atentan contra la diversificación productiva, sin descartar que los productores debieran romper paradigmas y salir de su zona de confort, pues solo así podrán dar el salto en cuanto a la diversificación y crecimiento de sus unidades de producción. Al respecto, algo que quedó al descubierto con el trabajo es que quizás las políticas públicas deban modificar su manera de incentivar a los productores, e invertir más en apoyos de acompañamiento a los productores, en lugar de recursos económicos para la producción, los cuales debieran seguirse brindando, pero en menores proporciones.

Es importante para la universidad como institución el coadyuvar en el desarrollo local de los municipios; la Universidad de la Sierra no está exenta a este proceso. De aquí la importancia de contar con una buena relación con los gobiernos locales e implementar en forma conjunta programas sociales, económicos, culturales que ayuden al desarrollo de las regiones.

Por ello es necesario implementar estrategias que deriven de planes medibles y que signifiquen impactos directos en la transformación social, económica, y de cambio de cultura ambiental. La Universidad de la Sierra mediante su personal docente y estudiantes puede enfocar sus diversos proyectos acordes a las estrategias que se plantean, de tal forma que cada uno de ellos signifique cumplir con objetivos específicos.

Así mismo, es importante la búsqueda del desarrollo económico, sociocultural y políticos de la región de la sierra de Sonora, para con ello incrementar el nivel de vida de las familias sonorenses, independientemente de que conlleva al desarrollo del Estado. Hoy en día el acrecentamiento de la población mundial ha sido determinante para tomar en cuenta situaciones en las que antes no se pensaba, específicamente el deterioro de los recursos naturales que el ser humano lleva a cabo, esto debido a, que sus necesidades van en aumento gradualmente; ahora bien la Universidad de la Sierra debe formar especialistas que busquen la sustentabilidad de las regiones, es decir, aprender a trabajar con los recursos, sin que se vean afectados, para las futuras generaciones puedan disfrutar de los mismos.
Sabemos que buscar el desarrollo sustentable perecería ser una utopía, sin embargo, creemos que lo más importante, como IES, es iniciar procesos propositivos e integrales que logren hacer transformaciones sociales y económicas importantes, desde una perspectiva socio ambiental, administradas estratégicamente, siempre cuidando las costumbres y formas de pensar de los habitantes de la zona de estudio.

En la actualidad, el sector turístico incrementa de manera constante su dinamismo, y genera en si un ambiente de competencia y competitividad para quienes participan en él. Por lo tanto, los destinos turísticos requieren de ciertas estrategias para brindar y mantener la calidad y seguridad en los servicios que brindan a los turistas; dentro de estas acciones se encuentra la de asegurar que los prestadores de servicios turísticos cumplan con las disposiciones por las que fueron constituidas.

\section{BIBLIOGRAFÍA}

Ascanio, G. A. y Vinicius, C. M. (2009). Turismo Sustentable, el equilibrio necesario en el siglo XXI. México: Editorial Trillas.

Apodaca-González, C., Juárez-Sánchez, J. P., RamírezValverde, B. \& Figueroa Sterquel, R. (2014). Revitalización de fincas cafetaleras por medio del turismo rural: caso del municipio Coatepec, Veracruz. Revista Mexicana de Ciencias Agrícolas, (9) 1523-1535. Recuperado de http://2011.www.redalyc.org/articulo. oa? id $=263137781001$

COFETUR (2015). Ruta Sierra Alta. Recuperado de http:// www.sonoraturismo.gob.mx/rutasierraalta.php

Cuéntame INEGI Población, 2010, Población rural y urbana. Recuperado de:http://cuentame.inegi.org.mx/ poblacion/rur_urb.aspx?tema $=P$

Escobedo Garridō, J. S. (2014). El turismo rural, un desafío para pequeños agricultores. Revista Mexicana de Ciencias Agrícolas, (9) 1601-1613. Recuperado de http://www. redalyc.org/articulo.oa? $\mathrm{id}=263137781007$

Henríquez, C., Zechner, T. C. y Cioce Sampaio, C. A. (2010). Turismo y sus interacciones en las transformaciones del espacio rural. Revista Austral de Ciencias Sociales, (18) 21-31. Recuperado de http://www.redalyc.org/articulo. oa?id=45920743002

Hernández Sampieri, R., Fernández Collado, C., \& Baptista Lucio, P. (6a. ed.). (2014). Metodología de la Investigación. México: McGraw-Hill.

Méndez, M. (2002). Problemas económicos de México. México: Mc Graw Hill.

Porter, M. (1991). Estrategias Competitiva- Técnica para el Análisis de los Sectores Industriales y de la Competencia. México: CECSA. 
Secretaría de Turismo. (2002). Serie Turismo Secretaría de Turismo. (2004). Serie turismo Alternativo "Turismo Alternativo, una Alternativo "Cómo desarrollar un proyecto nueva forma de hacer Turismo". México. de Ecoturismo". México. 